\title{
Black hole interior mass formula
}

\author{
Parthapratim Pradhan ${ }^{\mathrm{a}}$ \\ Department of Physics, Vivekananda Satabarshiki Mahavidyalaya, Manikpara, West Midnapur 721513, West Bengal, India
}

Received: 1 February 2014 / Accepted: 4 May 2014 / Published online: 21 May 2014

(C) The Author(s) 2014. This article is published with open access at Springerlink.com

\begin{abstract}
We argue by explicit computations that, although the area product, horizon radii product, entropy product, and irreducible mass product of the event horizon and Cauchy horizon are universal, the surface gravity product, the surface temperature product and the Komar energy product of the said horizons do not seem to be universal for Kerr-Newman black hole spacetimes. We show the black hole mass formula on the Cauchy horizon following the seminal work by Smarr [Phys Rev Lett 30:71 (1973), Phys Rev D 7:289 (1973)] for the outer horizon. We also prescribe the four laws of black hole mechanics for the inner horizon. A new definition of the extremal limit of a black hole is discussed.
\end{abstract}

\section{Introduction}

An intriguing feature of stationary axially symmetric black holes is that the product of the horizon areas are often independent of the mass of the black hole. Rather such products depend on the charge and angular momentum of the black hole. They may also be formulated in terms of the proper radii of the Cauchy horizon and event horizon.

It is also known that every regular axisymmetric and stationary spacetime of an Einstein-Maxwell system with surrounding matter has a regular Cauchy horizon $\left(\mathcal{H}^{-}\right)$inside the event horizon $\left(\mathcal{H}^{+}\right)$if and only if the angular momentum $J$ and charge $Q$ do not both vanish. In contrast, the Cauchy horizon becomes singular and approaches a curvature singularity in the limit $J \rightarrow 0, Q \rightarrow 0$ [3-5].

The presence of the Cauchy horizon implies that in Boyer-Lindquist coordinates, the stationary and axisymmetric Einstein-Maxwell electro-vacuum equations are hyperbolic in nature in the interior vicinity of $\mathcal{H}^{+}$. The two horizons $\mathcal{H}^{+}$and $\mathcal{H}^{-}$describe the future and past boundary of this hyperbolic region. Remarkably, if the inner Cauchy horizon exists (i.e. if $J$ and $Q$ do not vanish simultaneously), then

a e-mail: pppradhan5@ rediffmail.com; pppradhan77@ gmail.com the product of the area $A_{ \pm}$of the horizons $\mathcal{H}^{ \pm}$for the $\mathrm{KN}$ family are expressed by the relation [3-5]

$\mathcal{A}_{+} \mathcal{A}_{-}=(8 \pi)^{2}\left(J^{2}+\frac{Q^{4}}{4}\right)$,

which is remarkably independent of the mass $(\mathcal{M}) . J$ and $Q$ are the angular momentum and charge of the black hole, respectively.

From the various string theoretic models and holographic principle followed by observations it is suggested that the product of certain Killing horizon areas is in fact independent of the black hole mass. From the idea of statistical mechanics based on microscopic models counting BPS states it is determined that this product of areas is sometimes quantized. Thus in the super-symmetric extremal limit, one obtains [6-12]

$\mathcal{A}_{+} \mathcal{A}_{-}=\left(8 \pi \ell_{p l}^{2}\right)^{2} N, \quad N \in \mathbb{N}$,

where $\ell_{p l}$ is the Planck length. When one moves away from the extremality and super-symmetry, the area product is discretized [13] in terms of the Planck area and the fine structure constant, i.e.

$\mathcal{A}_{+} \mathcal{A}_{-}=\left(8 \pi \ell_{p l}\right)^{2}\left[\ell(\ell+1)+\frac{\alpha^{2} q^{2}}{4}\right]$,

which implies that the quantization rules break down, only because the fine structure constant $(\alpha)$ is not an integer. Here $\ell \in \mathbb{N}$ and $q \in \mathbb{Z}$.

The fact is that the Cauchy horizon is an "infinite blueshift" region and classically unstable due to the linear perturbation. Thus when an observer crosses the Cauchy horizon $r=r_{-}$, he/she observes all of the events which occur at "Region-I" and also the electromagnetic and gravitational field oscillations at infinite frequency are seen which actually occur at finite frequency in "Region-I" [14].

Despite the above fact, the Cauchy horizon is an interesting venue where we study the following features of the charged rotating spacetimes and rotating spacetimes. 
- We prove in Sect. 2, like the area and entropy product, that the surface gravity product, surface temperature or black hole temperature product and the Komar energy product of both inner horizon and outer horizon do not show any global properties due to the mass dependence. Such products are not universal in nature.

- We explicitly show in Sect. 3 that the black hole mass or ADM mass can be expressed in terms of the area of the Cauchy horizon $\left(\mathcal{H}^{-}\right)$:

$\mathcal{M}^{2}=\frac{\mathcal{A}_{-}}{16 \pi}+\frac{4 \pi J^{2}}{\mathcal{A}_{-}}+\frac{Q^{2}}{2}+\frac{\pi Q^{4}}{\mathcal{A}_{-}}$,

and we prove that the mass can be expressed as a sum of the surface energy, the rotational energy, and the electromagnetic energy of the Cauchy horizon $\left(\mathcal{H}^{-}\right)$:

$\mathcal{M}=\mathcal{E}_{s-}+\mathcal{E}_{r-}+\mathcal{E}_{\text {em- }}$.

- Also we find in Sect. 4 that the Christodoulou-Ruffini [15] mass formula may be expressed in terms of the area of the Cauchy horizon $\left(\mathcal{H}^{-}\right)$:

$\mathcal{M}^{2}=\left(\mathcal{M}_{\text {irr- }}+\frac{Q^{2}}{4 \mathcal{M}_{\text {irr- }}}\right)^{2}+\frac{J^{2}}{4\left(\mathcal{M}_{\text {irr- }}\right)^{2}}$.

- We further investigate the laws of black hole mechanics for the inner horizon in Sect. 5.

- We also point out that the product of Christodoulou's irreducible mass of the inner horizon (Cauchy horizon) and the outer horizon (event horizon) are independent of the mass, i.e.,

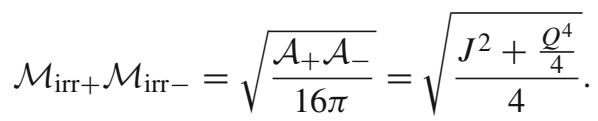

- We also shortly derive the identity $K_{\chi^{\mu}-}=2 \mathcal{S}_{-} T_{-}$on the Cauchy horizon in Sect. 6.

- The entropy of the Cauchy horizon may be expressed in the form $\mathcal{S}_{-}=\frac{E_{-}}{2 T_{-}}$as described in Sect. 7.

\section{Charged rotating black hole}

The complete gravitational collapse of a charged body always produces a Kerr-Newman $(\mathrm{KN})$ black hole [16], which is the most general class among the classical black hole solutions. It also is uniquely described by the electro-vacuum black hole solutions of the Einstein-Maxwell system. It can be specified by three parameters: the black hole mass $\mathcal{M}$, the charge $Q$, and the angular momentum per unit mass $a=J / \mathcal{M}$. As long as $\mathcal{M}^{2} \geq Q^{2}+a^{2}$ the $\mathrm{KN}$ metric describes a black hole, otherwise it has a naked ringlike singularity. It possesses two horizons, namely the event horizon $\left(\mathcal{H}^{+}\right)$or outer horizon and the Cauchy horizon $\left(\mathcal{H}^{-}\right)$or inner horizon. The proper radii of event horizon and Cauchy horizon are

$r_{ \pm}=\mathcal{M} \pm \sqrt{\mathcal{M}^{2}-a^{2}-Q^{2}}$ and $r_{+}>r_{-}$,

whose product is

$r_{+} r_{-}=a^{2}+Q^{2}$.

It is speculated that it does not depend on the mass but depends on the charge and the Kerr parameter [17].

Then the areas $[18,19]$ of the two horizons $\left(\mathcal{H}^{ \pm}\right)$are

$\mathcal{A}_{ \pm}=\iint \sqrt{g_{\theta \theta} g_{\phi \phi}}=4 \pi\left(r_{ \pm}^{2}+a^{2}\right)$.

The angular velocities of $\mathcal{H}^{ \pm}$are

$\Omega_{ \pm}=\frac{a}{r_{ \pm}^{2}+a^{2}}$.

The semiclassical Bekenstein-Hawking entropy of $\mathcal{H}^{ \pm}$ reads (in units in which $G=\hbar=c=1$ )

$\mathcal{S}_{ \pm}=\frac{\mathcal{A}_{ \pm}}{4}=\pi\left(r_{ \pm}^{2}+a^{2}\right)$.

The surface gravity of $\mathcal{H}^{ \pm}$is

$\kappa_{ \pm}=\frac{r_{ \pm}-r_{\mp}}{2\left(r_{ \pm}^{2}+a^{2}\right)}$ and $\kappa_{+}>\kappa_{-}$,

and the black hole temperature or Hawking temperature of $\mathcal{H}^{ \pm}$reads

$T_{ \pm}=\frac{\kappa_{ \pm}}{2 \pi}=\frac{r_{ \pm}-r_{\mp}}{4 \pi\left(r_{ \pm}^{2}+a^{2}\right)}$.

It should be noted that the event horizon is hotter than the Cauchy horizon i.e. $T_{+}>T_{-}$.

The Komar [20] energy for $\mathcal{H}^{ \pm}$is given by (as we will discuss elaborately in Sect. 7)

$E_{ \pm}=2 \mathcal{S}_{ \pm} T_{ \pm}= \pm \sqrt{\mathcal{M}^{2}-a^{2}-Q^{2}}$.

Finally the horizon Killing vector fields may be defined for $\mathcal{H}^{ \pm}$:

$\chi_{ \pm}^{a}=\left(\partial_{t}\right)^{a}+\Omega_{ \pm}\left(\partial_{\phi}\right)^{a}$.

If, in addition, the black hole is non-extremal (i.e., if there exists a trapped surface interior of the outer horizon) then the following relations hold:

$\mathcal{A}_{+}>\sqrt{(8 \pi)^{2}\left(J^{2}+\frac{Q^{4}}{4}\right)}>\mathcal{A}_{-}$.

Also the entropy product is

$\mathcal{S}_{+} \mathcal{S}_{-}=(2 \pi)^{2}\left(J^{2}+\frac{Q^{4}}{4}\right)$. 
It is also independent of mass $(\mathcal{M})$. The entropy of the nonextremal case satisfies the following inequality:

$\mathcal{S}_{+}>\sqrt{(2 \pi)^{2}\left(J^{2}+\frac{Q^{4}}{4}\right)}>\mathcal{S}_{-}$.

Similarly we can compute the product of surface gravities of $\mathcal{H}^{ \pm}$:

$\kappa_{+} \kappa_{-}=-\frac{\left(r_{+}-r_{-}\right)^{2}}{4\left(r_{+}^{2}+a^{2}\right)\left(r_{-}^{2}+a^{2}\right)}=-\frac{\mathcal{M}^{2}-a^{2}-Q^{2}}{\left(r_{+}^{2}+a^{2}\right)\left(r_{-}^{2}+a^{2}\right)}$.

The product of the surface temperatures of $\mathcal{H}^{ \pm}$reads

$$
\begin{aligned}
T_{+} T_{-} & =-\frac{\left(r_{+}-r_{-}\right)^{2}}{(4 \pi)^{2}\left(r_{+}^{2}+a^{2}\right)\left(r_{-}^{2}+a^{2}\right)} \\
& =-\frac{\mathcal{M}^{2}-a^{2}-Q^{2}}{(2 \pi)^{2}\left(r_{+}^{2}+a^{2}\right)\left(r_{-}^{2}+a^{2}\right)},
\end{aligned}
$$

and the product of the Komar energies of $\mathcal{H}^{ \pm}$is

$E_{+} E_{-}=\left(2 \mathcal{S}_{+} T_{+}\right)\left(2 \mathcal{S}_{-} T_{-}\right)=-\left(\mathcal{M}^{2}-a^{2}-Q^{2}\right)$.

It seems that these products are not universal.

In case of pure Einstein gravity (without Maxwell field) the above relations are reduced to the following. For the proper radii product of $\mathcal{H}^{ \pm}$:

$r_{+} r_{-}=a^{2}$.

For the area product of $\mathcal{H}^{ \pm}$:

$\mathcal{A}_{+} \mathcal{A}_{-}=(8 \pi J)^{2}$.

For the entropy product of $\mathcal{H}^{ \pm}$:

$\mathcal{S}_{+} \mathcal{S}_{-}=(2 \pi J)^{2}$.

For the surface gravity product of $\mathcal{H}^{ \pm}$:

$\kappa_{+} \kappa_{-}=-\frac{\left(r_{+}-r_{-}\right)^{2}}{4\left(r_{+}^{2}+a^{2}\right)\left(r_{-}^{2}+a^{2}\right)}=-\frac{\mathcal{M}^{2}-a^{2}}{\left(r_{+}^{2}+a^{2}\right)\left(r_{-}^{2}+a^{2}\right)}$.

For the temperature product of $\mathcal{H}^{ \pm}$:

$$
\begin{aligned}
T_{+} T_{-} & =-\frac{\left(r_{+}-r_{-}\right)^{2}}{(4 \pi)^{2}\left(r_{+}^{2}+a^{2}\right)\left(r_{-}^{2}+a^{2}\right)} \\
& =-\frac{\mathcal{M}^{2}-a^{2}}{(2 \pi)^{2}\left(r_{+}^{2}+a^{2}\right)\left(r_{-}^{2}+a^{2}\right)} .
\end{aligned}
$$

For the Komar energy product of $\mathcal{H}^{ \pm}$:

$E_{+} E_{-}=\left(2 \mathcal{S}_{+} T_{+}\right)\left(2 \mathcal{S}_{-} T_{-}\right)=-\left(\mathcal{M}^{2}-a^{2}\right)$.

So the product of the area and entropy of the two horizons are proportional to the square of the spin parameter $J$. Surface gravity product, surface temperature product, and Komar energy product depend on the mass. Thus we may conclude that they are not universal except the area product and entropy product.

\section{Smarr formula for Cauchy horizon $\left(\mathcal{H}^{-}\right)$}

In the original paper by Larry Smarr $[1,2]$ the area for the charged rotating black hole is described by the following relation:

$\mathcal{A}=4 \pi\left(2 \mathcal{M}^{2}-Q^{2}+2 \sqrt{\mathcal{M}^{4}-J^{2}-\mathcal{M}^{2} Q^{2}}\right)$.

It is indeed constant over the exterior horizon. We suggest here that there are two horizons, so correspondingly both areas must be constant i.e. the area can be expressed as

$\mathcal{A}_{ \pm}=4 \pi\left(2 \mathcal{M}^{2}-Q^{2} \pm 2 \sqrt{\mathcal{M}^{4}-J^{2}-\mathcal{M}^{2} Q^{2}}\right)$.

Inverting the above relation one can obtain the black hole mass or ADM mass, which can be expressed in terms of the areas of both horizons $\mathcal{H}^{ \pm}$,

$\mathcal{M}^{2}=\frac{\mathcal{A}_{ \pm}}{16 \pi}+\frac{4 \pi J^{2}}{\mathcal{A}_{ \pm}}+\frac{Q^{2}}{2}+\frac{\pi Q^{4}}{\mathcal{A}_{ \pm}}$.

It is remarkable that the mass can be expressed in terms of both the area of $\mathcal{H}^{+}$and of $\mathcal{H}^{-}$. Now what happens with the mass differential? It is indeed expressed as three physical invariants of both $\mathcal{H}^{+}$and $\mathcal{H}^{-}$,

$\mathrm{d} \mathcal{M}=\mathcal{T}_{ \pm} \mathrm{d} \mathcal{A}_{ \pm}+\Omega_{ \pm} \mathrm{d} J+\Phi_{ \pm} \mathrm{d} Q$,

where

$\mathcal{T}_{ \pm}=\frac{1}{\mathcal{M}}\left(\frac{1}{32 \pi}-\frac{2 \pi J^{2}}{\mathcal{A}_{ \pm}^{2}}-\frac{\pi Q^{4}}{2 \mathcal{A}_{ \pm}^{2}}\right)$

$\Omega_{ \pm}=\frac{4 \pi J}{\mathcal{M} \mathcal{A}_{ \pm}}$

$\Phi_{ \pm}=\frac{1}{\mathcal{M}}\left(\frac{Q}{2}+\frac{2 \pi Q^{3}}{\mathcal{A}_{ \pm}}\right)$,

where

$\mathcal{T}_{ \pm}=$Effective surface tension for $\mathcal{H}^{+}$and $\mathcal{H}^{-}$

$\Omega_{ \pm}=$Angular velocity for $\mathcal{H}^{ \pm}$

$\Phi_{ \pm}=$Electromagnetic potentials for $\mathcal{H}^{ \pm}$.

The effective surface tension may be rewritten as

$$
\begin{aligned}
\mathcal{T}_{ \pm} & =\frac{1}{\mathcal{M}}\left(\frac{1}{32 \pi}-\frac{2 \pi J^{2}}{\mathcal{A}_{ \pm}^{2}}-\frac{\pi Q^{4}}{2 \mathcal{A}_{ \pm}^{2}}\right) \\
& =\frac{1}{32 \pi \mathcal{M}}\left(1-\frac{16 \pi^{2}\left(4 J^{2}+Q^{4}\right)}{\mathcal{A}_{ \pm}^{2}}\right) \\
& =\frac{1}{16 \pi \mathcal{M}}\left(1-\frac{\left(2 \mathcal{M}^{2}-Q^{2}\right)}{r_{ \pm}^{2}+a^{2}}\right)
\end{aligned}
$$




$$
\begin{aligned}
& = \pm \frac{\sqrt{\mathcal{M}^{2}-a^{2}-Q^{2}}}{8 \pi\left(r_{ \pm}^{2}+a^{2}\right)} \\
& =\frac{r_{ \pm}-\mathcal{M}}{8 \pi\left(r_{ \pm}^{2}+a^{2}\right)}=\frac{\kappa_{ \pm}}{8 \pi}
\end{aligned}
$$

where $\kappa_{ \pm}$are the surface gravities of $\mathcal{H}^{ \pm}$as previously defined.

Thus the mass can be expressed in terms of these quantities both for $\mathcal{H}^{ \pm}$in the simple bilinear form

$\mathcal{M}=2 \mathcal{T}_{ \pm} \mathcal{A}_{ \pm}+2 J \Omega_{ \pm}+\Phi_{ \pm} Q$.

This has been derived from the homogeneous function of degree $\frac{1}{2}$ in $\left(\mathcal{A}_{ \pm}, J, Q^{2}\right)$. Remarkably, $\mathcal{T}_{ \pm}, \Omega_{ \pm}$and $\Phi_{ \pm}$can be defined and are constant on $\mathcal{H}^{+}$and $\mathcal{H}^{-}$for any stationary, axially symmetric spacetime. Since $d \mathcal{M}$ is a total differential, one may choose freely any path of integration in $\left(\mathcal{A}_{ \pm}, J, Q\right)$ space. Thus the surface energy $\mathcal{E}_{s \pm}$ for $\mathcal{H}^{+}$and $\mathcal{H}^{-}$can be defined by

$\mathcal{E}_{S \pm}=\int_{0}^{\mathcal{A}_{ \pm}} \mathcal{T}\left(\tilde{\mathcal{A}_{ \pm}}, 0,0\right) \mathrm{d} \tilde{\mathcal{A}_{ \pm}}$

the rotational energy for $\mathcal{H}^{+}$and $\mathcal{H}^{-}$can be defined by

$\mathcal{E}_{r \pm}=\int_{0}^{J} \Omega_{ \pm}\left(\mathcal{A}_{ \pm}, \tilde{J}, 0\right) \mathrm{d} \tilde{J}, \quad \mathcal{A}_{ \pm}$fixed

and the electromagnetic energies for $\mathcal{H}^{+}$and $\mathcal{H}^{-}$are

$\mathcal{E}_{\text {em }}=\int_{0}^{Q} \Phi_{ \pm}\left(\mathcal{A}_{ \pm}, J, \tilde{Q}\right) \mathrm{d} \tilde{Q}, \quad \mathcal{A}_{ \pm}, J$ fixed.

We may rewrite Eq. (41) as

$\mathcal{M}=\frac{\kappa_{ \pm}}{4 \pi} \mathcal{A}_{ \pm}+2 J \Omega_{ \pm}+\Phi_{ \pm} Q$

or

$\mathcal{M}-2 J \Omega_{ \pm}-\Phi_{ \pm} Q=\frac{\kappa_{ \pm}}{4 \pi} \mathcal{A}_{ \pm}$,

or

$\mathcal{M}-2 J \Omega_{ \pm}-\Phi_{ \pm} Q=\frac{T_{ \pm}}{2} \mathcal{A}_{ \pm}$,

or

$\frac{\mathcal{M}}{2}=T_{ \pm} \mathcal{S}_{ \pm}+J \Omega_{ \pm}+\frac{\Phi_{ \pm} Q}{2}$.

This is recognized as a generalized Smarr-Gibbs-Duhem relation for $\mathcal{H}^{ \pm}$. Here ' + ' indicates the event horizon, which was already discussed in the literature [21]. We derive the above relation here for the Cauchy horizon only and for the record we also mention here the two horizons.

Now we define a new parameter set $\left(\eta_{ \pm}, \beta_{ \pm}, \epsilon_{ \pm}\right)$which is related to the quantities $\left(\mathcal{A}_{ \pm}, J, Q\right)$ to study the intrinsic geometry for the Cauchy horizon $\left(\mathcal{H}^{-}\right)$of a charged rotating black hole; it is given by

$\eta_{ \pm}=\sqrt{r_{ \pm}^{2}+a^{2}}=\sqrt{\frac{\mathcal{A}_{ \pm}}{4 \pi}}$,

$\beta_{ \pm}=\frac{a}{\sqrt{r_{ \pm}^{2}+a^{2}}}=\frac{a}{\eta_{ \pm}}$

$\epsilon_{ \pm}=\frac{Q}{\eta_{ \pm}}$

Therefore the integrated mass formula for $\mathcal{H}^{ \pm}$is found to be

$$
\begin{aligned}
\mathcal{M} & =\frac{\eta_{ \pm}\left(1+\epsilon_{ \pm}^{2}\right)}{2 \sqrt{1-\beta_{ \pm}^{2}}}, \\
\mathcal{E}_{s \pm} & =\frac{\eta_{ \pm}}{2}, \\
\mathcal{E}_{r \pm} & =\frac{\eta_{ \pm}}{2}\left[\frac{1}{\sqrt{1-\beta_{ \pm}^{2}}}-1\right], \\
\mathcal{E}_{e m \pm} & =\frac{\eta_{ \pm} \epsilon_{ \pm}^{2}}{2 \sqrt{1-\beta_{ \pm}^{2}}},
\end{aligned}
$$

with

$\mathcal{M}=\mathcal{E}_{s \pm}+\mathcal{E}_{r \pm}+\mathcal{E}_{\text {em }}$.

Interestingly mass can be expressed as a sum of surface energy, rotational energy, and electromagnetic energy of the two horizons $\mathcal{H}^{ \pm}$. We have already seen the above discussion in [2] for the event horizon. We derive here the above relation for the Cauchy horizon only and for the sake of completeness we mention both cases.

\section{Christodoulou's irreducible mass for Cauchy horizon}

Besides the black hole event horizon, there exists a second horizon inside the black hole-the Cauchy horizon or inner horizon $\left(\mathcal{H}^{-}\right)$. It is defined as the future boundary of the domain of dependence of the $\left(\mathcal{H}^{+}\right)$. What happens with the Christodoulou-Ruffini [15] mass formula for the Cauchy horizon? This is an important issue, which we will discuss now. The irreducible mass is defined as

$\mathcal{M}_{\text {irr } \pm}=\frac{\sqrt{r_{ \pm}^{2}+a^{2}}}{2}=\sqrt{\frac{\mathcal{A}_{ \pm}}{16 \pi}}$,

where + indicates $\mathcal{H}^{+}$and - indicates $\mathcal{H}^{-}$. The area and angular velocity can be expressed in terms of $\mathcal{M}_{\text {irr } \pm}$ :

$\mathcal{A}_{ \pm}=16 \pi\left(\mathcal{M}_{\text {irr } \pm}\right)^{2}=4 \pi \rho_{ \pm}^{2}$

and

$\Omega_{ \pm}=\frac{a}{r_{ \pm}^{2}+a^{2}}=\frac{a}{4\left(\mathcal{M}_{\mathrm{irr} \pm}\right)^{2}}$. 
Interestingly, the products of the irreducible mass of $\mathcal{H}^{ \pm}$are universal.

$$
\begin{aligned}
\mathcal{M}_{\text {irr }+} \mathcal{M}_{\text {irr }-} & =\sqrt{\frac{\mathcal{A}_{+} \mathcal{A}_{-}}{16 \pi}} \\
& =\sqrt{\frac{J^{2}+\frac{Q^{4}}{4}}{4}} .
\end{aligned}
$$

The rest mass of a rotating charged black hole is defined by the Christodoulou-Ruffini mass formula in terms of its irreducible mass and its angular momentum $(J)$ and charge $(Q)$ by the formula

$$
\begin{aligned}
\mathcal{M}^{2} & =\left(\mathcal{M}_{\text {irr } \pm}+\frac{Q^{2}}{4 \mathcal{M}_{\text {irr } \pm}}\right)^{2}+\frac{J^{2}}{4\left(\mathcal{M}_{\text {irr } \pm}\right)^{2}} . \\
& =\left(\mathcal{M}_{\text {irr } \pm}+\frac{Q^{2}}{2 \rho_{ \pm}}\right)^{2}+\left(\frac{J}{\rho_{ \pm}}\right)^{2} \\
& =\left(m_{r \pm}\right)^{2}+\left(p_{ \pm}\right)^{2} \\
& =\left(\gamma_{ \pm} m_{r \pm}\right)^{2}
\end{aligned}
$$

where $p_{ \pm}=\frac{J}{\rho_{ \pm}}$is an effective momentum and the effective rest mass $m_{r \pm}$ can be defined as

$m_{r \pm}=\mathcal{M}_{\text {irr } \pm}+\frac{Q^{2}}{2 \rho_{ \pm}}=\mathcal{M}_{\text {irr } \pm}+\frac{Q^{2}}{4 \mathcal{M}_{\text {irr } \pm}}$.

Also the corresponding gamma factor is given by

$$
\gamma_{ \pm}=\frac{1}{\sqrt{1-v_{ \pm}^{2}}}=\frac{1}{\sqrt{1-\frac{a^{2}}{4\left(\mathcal{M}_{\mathrm{irr} \pm}\right)^{2}}}} .
$$

It may be noted that $\left(v_{ \pm}\right)^{2}$ is a strange product of the angular velocity and angular momentum (the conjugate momentum variable) divided by the mass of the black hole,

$\left(v_{ \pm}\right)^{2}=a \Omega_{ \pm}=\frac{J}{M} \Omega_{ \pm}$.

One may compare various formulas for the black hole quantities and certain formulas from mechanics and electromagnetism by rewriting the formula either in $\mathcal{M}_{\text {irr } \pm}$ or $\rho_{ \pm}$in geometric units, which corresponds to a mass or length variable.

A reversible process is characterized by an unchanged irreducible mass, whereas an irreversible process is characterized by an increase in irreducible mass of a black hole. It should be noted that there exists no process which will decrease the $\mathcal{M}_{\text {irr }}$ for a Cauchy horizon.

This mass decomposes into an irreducible mass $\mathcal{M}_{\text {irr }}$ and a rotational energy $\mathcal{M}-\mathcal{M}_{\text {irr }}$ for a Kerr black hole as shown by Christodoulou [22]. For a Kerr-Newman black hole the mass can be written as both for $\mathcal{H}^{+}$and $\mathcal{H}^{-}$in the small angular momentum limit, and one has

$\mathcal{M}=\mathcal{M}_{\text {irr } \pm}+\frac{Q^{2}}{4 \mathcal{M}_{\text {irr } \pm}}+\mathcal{K}_{ \pm}$, where

$\mathcal{K}_{ \pm}=\frac{1}{2}\left(\mathcal{M}_{\text {irr } \pm}+\frac{Q^{2}}{4 \mathcal{M}_{\text {irr }}}\right)\left(v_{ \pm}\right)^{2}$.

This looks like the expression for the kinetic energy in classical mechanics. The effective speed $v_{ \pm}$is given by

$v_{ \pm}=\frac{p_{ \pm}}{\mathcal{M}}=\frac{J}{\mathcal{M} \rho_{ \pm}}=\frac{a}{\rho_{ \pm}}=\frac{a}{2 \mathcal{M}_{\text {irr } \pm}}=\rho_{ \pm} \Omega_{ \pm}$,

where $\rho_{ \pm}=\sqrt{r_{ \pm}^{2}+a^{2}}=2 \mathcal{M}_{\text {irr } \pm \text {. Thus Eq. (70) reduces to }}$

$\mathcal{K}_{ \pm}=\frac{1}{2} m_{r \pm}\left(v_{ \pm}\right)^{2}=\frac{J^{2}}{2 I_{ \pm}}=\frac{1}{2} I_{ \pm} \Omega_{ \pm}^{2}$,

where $I_{ \pm}=m_{r \pm}\left(\rho_{ \pm}\right)^{2}$ plays the role of a moment of inertia in this limit. The above discussion for event horizon can be found in [23]. We have derived the above formula here for Cauchy horizon only.

When the Penrose process [24,25] is taken into account, this leads to the following exact differential relationship between mass and angular momentum, which is also characterized by reversible transformations, as described by Christodoulou and Ruffini:

$\mathrm{d} \mathcal{M}=\frac{a \mathrm{~d} J+r_{ \pm} Q \mathrm{~d} Q}{r_{ \pm}^{2}+a^{2}}$.

After integration we obtain the Christodoulou and Ruffini mass formula (62), when the following condition is satisfied:

$\frac{J^{2}}{4\left(\mathcal{M}_{\text {irr } \pm}\right)^{2}}+\frac{Q^{4}}{16\left(\mathcal{M}_{\text {irr } \pm}\right)^{4}} \leq 1$.

\section{The four laws of black hole mechanics on the event horizon $\left(\mathcal{H}^{+}\right)$and Cauchy horizon $\left(\mathcal{H}^{-}\right)$}

Following the remarkable discovery by Carter et al. [26], we reformulate the black hole thermodynamics both for the event horizon and the Cauchy horizon, which is analogous to the classical laws of thermodynamics as follows:

- The Zeroth Law: The surface gravity, $\kappa_{ \pm}$of a stationary black hole is constant over both the event horizon $\left(\mathcal{H}^{+}\right)$ and the Cauchy horizon $\left(\mathcal{H}^{-}\right)$, respectively.

- The First Law: Any perturbation of a stationary black holes, a change of mass (change of energy), is related to a change of mass, angular momentum, and electric charge by

$$
\mathrm{d} \mathcal{M}=\frac{\kappa_{ \pm}}{8 \pi} \mathrm{d} \mathcal{A}_{ \pm}+\Omega_{ \pm} \mathrm{d} J+\Phi_{ \pm} \mathrm{d} Q
$$

It can be seen that $\frac{\kappa_{ \pm}}{8 \pi}$ is analogous to the temperature of $\mathcal{H}^{ \pm}$in the same way as $\mathcal{A}_{ \pm}$is analogous to entropy. 
It should be noted that $\frac{\kappa_{ \pm}}{8 \pi}$ and $\mathcal{A}_{ \pm}$are distinct from the temperature and entropy of the black hole.

The above expression $\frac{\kappa_{ \pm}}{8 \pi}$ can be derived from Eq. (35) in the following way.

The effective surface tension can be rewritten as

$$
\mathcal{T}_{ \pm}=\frac{\kappa_{ \pm}}{8 \pi}=\frac{\partial \mathcal{M}}{\partial \mathcal{A}_{ \pm}}
$$

and

$$
\begin{aligned}
& \Omega_{ \pm}=\frac{4 \pi J}{\mathcal{M} \mathcal{A}_{ \pm}}=\frac{\partial \mathcal{M}}{\partial J} \\
& \Phi_{ \pm}=\frac{1}{\mathcal{M}}\left(\frac{Q}{2}+\frac{2 \pi Q^{3}}{\mathcal{A}_{ \pm}}\right)=\frac{\partial \mathcal{M}}{\partial Q} .
\end{aligned}
$$

- The Second Law: The area $\mathcal{A}_{ \pm}$of both event horizon $\left(\mathcal{H}^{+}\right)$ and Cauchy horizon $\left(\mathcal{H}^{-}\right)$never decreases, i.e.

$$
\mathrm{d} \mathcal{A}_{ \pm}=\frac{4 \mathcal{A}_{ \pm}}{r_{ \pm}-r_{\mp}}\left(\mathrm{d} \mathcal{M}-\boldsymbol{\Omega}_{ \pm} . \mathrm{d} \mathbf{J}-\Phi_{ \pm} \mathrm{d} Q\right) \geq 0
$$

or

$$
\mathrm{d} \mathcal{M}_{\mathrm{irr} \pm}=\frac{2 \mathcal{M}_{\mathrm{irr} \pm}}{r_{ \pm}-r_{\mp}}\left(\mathrm{d} \mathcal{M}-\boldsymbol{\Omega}_{ \pm} . \mathrm{d} \mathbf{J}-\Phi_{ \pm} \mathrm{d} Q\right) \geq 0 .
$$

The change in irreducible mass of both event horizon $\left(\mathcal{H}^{+}\right)$ and Cauchy horizon $\left(\mathcal{H}^{-}\right)$can never be negative.

It follows immediately from the above equation that

$$
\mathrm{d} \mathcal{M}>\boldsymbol{\Omega}_{ \pm} \cdot \mathrm{d} \mathbf{J}+\Phi_{ \pm} \mathrm{d} Q .
$$

- The Third Law: It is impossible by any mechanism, no matter how idealized, to reduce $\kappa_{ \pm}$, the surface gravity of both the event horizon $\left(\mathcal{H}^{+}\right)$and the Cauchy horizon $\left(\mathcal{H}^{-}\right)$, to zero by a finite sequence of operations.

\section{Komar conserved quantity $=$ 2 x entropy on $\mathcal{H}^{ \pm}$x temperature on $\mathcal{H}^{ \pm}$ or $K_{\chi}{ }^{\mu} \pm 2 \mathcal{S}_{ \pm} T_{ \pm}$}

It is well known [27] that on the $\mathcal{H}^{+}$the Komar conserved quantity $\left(K_{\chi^{\mu}+}\right)$ corresponding to a null Killing vector $\chi^{\mu}+$ is equal to twice the product of the entropy $\left(\mathcal{S}_{+}\right)$ on $\mathcal{H}^{+}$and the temperature $\left(T_{+}\right)$on $\mathcal{H}^{+}$. Here we shall derive a similar expression to the one that holds on $\mathcal{H}^{-}$. Thus we have to prove the identity $K_{\chi^{\mu}}=2 \mathcal{S}_{-} T_{-}$on the $\mathcal{H}^{-}$.

Due to the stationarity and axially symmetric nature of the Kerr-Newman spacetime, the spacetime has two Killing vectors. These two vectors are $\xi^{\mu}{ }_{(t)}=(1,0,0,0)$ and $\xi^{\mu}{ }_{(\phi)}=$ $(0,0,0,1)$, which corresponds to timelike and spacelike in the asymptotic limit. Thus we can define a Killing vector on both $\left(\mathcal{H}^{ \pm}\right)$which is a combination of these two vectors. We have

$\chi_{ \pm}^{\mu}=\xi_{(t)}^{\mu}+\Omega_{ \pm} \xi_{(\phi)}^{\mu}=\left(1,0,0, \Omega_{ \pm}\right)$.

It should be noted that on the $\left(\mathcal{H}^{ \pm}\right),\left.\chi^{\mu} \chi_{\mu}\right|_{r=r_{ \pm}}=0$; then $\xi^{\mu}$ becomes a null Killing vector. Now we can define a Komar conserved quantity on $\mathcal{H}^{ \pm}$, which corresponds to the Killing vectors being given by

$$
K_{\chi^{\mu} \pm}=K_{\xi^{\mu}(t)}+\Omega_{ \pm} K_{\xi^{\mu}(\phi)},
$$

where $K_{\chi^{\mu} \pm}$ is the Komar conserved quantity corresponding to the timelike Killing vector defined by

$K_{\xi^{\mu}(t)}=-\frac{1}{8 \pi} \int_{\partial \Sigma} * \mathrm{~d} \sigma$,

whose one form is given by

$\sigma=\xi_{(t) \mu} \mathrm{d} x^{\mu}=g_{t \mu} \mathrm{d} x^{\mu}=g_{t t} \mathrm{~d} t+g_{t \phi} \mathrm{d} \phi$,

and $* d \sigma$ is the dual to the two-form $d \sigma . d \Sigma$ is an appropriate boundary surface of a spatial three-volume $(\Sigma)$.

Similarly we can define the Komar conserved quantity on $\mathcal{H}^{ \pm}$corresponding to the spacelike Killing vector, given by

$K_{\xi^{\mu}(\phi)}=-\frac{1}{8 \pi} \int_{\partial \Sigma} * \mathrm{~d} \eta$,

where the spacelike Killing one-form is defined as

$\eta=\xi_{(\phi) \mu} \mathrm{d} x^{\mu}=g_{\mu \phi} \mathrm{d} x^{\mu}=g_{t \phi} \mathrm{d} t+g_{\phi \phi} \mathrm{d} \phi$.

After some computations we find

$K_{\chi^{\mu} \pm}=\mathcal{M}-2 \mathcal{M} \frac{a^{2}}{r_{ \pm}^{2}+a^{2}}-\frac{Q^{2} r_{ \pm}}{r_{ \pm}^{2}+a^{2}}$.

Using the expressions $\Omega_{ \pm}$and $r_{ \pm}$, we simplify the above equation rewriting it in the compact form

$$
\begin{aligned}
K_{\chi^{\mu} \pm} & = \pm \sqrt{\mathcal{M}^{2}-a^{2}-Q^{2}}=\frac{r_{ \pm}-r_{\mp}}{2} \\
& =2\left[\pi\left(r_{ \pm}^{2}+a^{2}\right)\right] \frac{r_{ \pm}-r_{\mp}}{4 \pi\left(r_{ \pm}^{2}+a^{2}\right)} \\
& =\frac{\mathcal{A}_{ \pm} \kappa_{ \pm}}{4 \pi}=2\left(\frac{\mathcal{A}_{ \pm}}{4}\right)\left(\frac{\kappa \pm}{2 \pi}\right) \\
& =2 \mathcal{S}_{ \pm} T_{ \pm} .
\end{aligned}
$$

Thus we have obtained on the $\mathcal{H}^{ \pm}$the Komar conserved charge corresponding to the null Killing vector, which is twice the product of the entropy and the surface temperature of the Kerr-Newman black hole. We can connect this quantity with a similar relation which has been derived in the previous section on the $\mathcal{H}^{ \pm}$. We have

$E_{ \pm}=2 \mathcal{S}_{ \pm} T_{ \pm}$ 
where $E_{ \pm}$is the Noether charge of the diffeomorphism symmetry on $\mathcal{H}^{ \pm}$. It should be noted that the above relation has been derived particularly for a static local Killing horizon [28], which may or may not be a black hole event horizon. However, a Kerr-Newman spacetime is stationary and therefore, whether it is valid for any stationary spacetime is not clear, but we have explicitly computed a similar relation on the Cauchy horizon $\mathcal{H}^{-}$. Introducing the scalar potential on the $\mathcal{H}^{ \pm}$, we have thus got

$\Phi_{ \pm}=\frac{Q r_{ \pm}}{r_{ \pm}^{2}+a^{2}}$.

Equation (88) can be rewritten in the form

$K_{\chi^{\mu} \pm}=\mathcal{M}-2 J \Omega_{ \pm}-\Phi_{ \pm} Q$.

Again from Eq. (41) we have

$$
\begin{aligned}
\mathcal{M}-2 J \Omega_{ \pm}-\Phi_{ \pm} Q & =\frac{\mathcal{A}_{ \pm} \kappa_{ \pm}}{4 \pi} \\
& =\frac{\mathcal{A}_{ \pm} T_{ \pm}}{2} .
\end{aligned}
$$

This is the well-known Smarr formula on $\mathcal{H}^{ \pm}$. In the literature we have seen the above discussion of the event horizon only. We extend the above relations, particularly for the Cauchy horizon. For the sake of completeness we also compute all the relations for the event horizon.

\section{Generalized Smarr formula for mass on the Cauchy horizon}

In this section we will derive for a stationary state black hole spacetime that the entropy can be expressed as $\mathcal{S}_{ \pm}=\frac{E_{ \pm}}{2 T_{ \pm}}$ on the $\mathcal{H}^{ \pm}$, where $T_{ \pm}$is the Hawking temperature on $\mathcal{H}^{ \pm}$ and $E_{ \pm}$is shown to be the Komar energy on the $\mathcal{H}^{ \pm}$. We also derive the generalized Smarr formula for a mass on $\mathcal{H}^{ \pm}$. This is compatible with the relation in Eq. (97). We already know from [29] that the Komar energy for a Kerr-Newman black hole in a compact form on the $\mathcal{H}^{+}$is

$$
\begin{aligned}
2 \mathcal{S}_{+} T_{+} & =E_{+}=\sqrt{\mathcal{M}^{2}-a^{2}-Q^{2}} \\
& =\mathcal{M}-\frac{Q^{2}}{r_{+}}-2 J \Omega_{+}\left(1-\frac{Q^{2}}{2 \mathcal{M} r_{+}}\right) \\
& =\mathcal{M}-2 J \Omega_{+}-Q V_{+},
\end{aligned}
$$

where $V_{+}=\frac{Q}{r_{+}}-\frac{J Q \Omega_{+}}{\mathcal{M} r_{+}}$. Similarly we can easily obtain on the Cauchy horizon the Komar energy for a Kerr-Newman black hole:

$2 \mathcal{S}_{-} T_{-}=E_{-}=-\sqrt{\mathcal{M}^{2}-a^{2}-Q^{2}}$.

Remarkably the energy is negative, which also reveals that the Killing vector field is negative inside $\mathcal{H}^{+}$. Thus the energy is negative on $\mathcal{H}^{-}$due to this fact.
In fact we can rewrite Eq. (101) as

$$
\begin{aligned}
2 \mathcal{S}_{-} T_{-} & =E_{-}=-\sqrt{\mathcal{M}^{2}-a^{2}-Q^{2}} \\
& =\mathcal{M}-2 J \Omega_{-}-Q V_{-},
\end{aligned}
$$

where $V_{-}=\frac{Q}{r_{-}}-\frac{J Q \Omega_{-}}{\mathcal{M} r_{-}}$.

Thus Eqs. (98) and (102) can be written on both the horizons $\left(\mathcal{H}^{ \pm}\right)$in a compact form as

$$
\begin{aligned}
2 \mathcal{S}_{ \pm} T_{ \pm} & =E_{ \pm}= \pm \sqrt{\mathcal{M}^{2}-a^{2}-Q^{2}} \\
& =\mathcal{M}-2 J \Omega_{ \pm}-Q V_{ \pm} .
\end{aligned}
$$

\section{Degenerate black hole or extremal black hole}

Thus one may define an extremal black hole as a black hole where the radii of event horizon and Cauchy horizon are converging i.e.,

$r_{+}=r_{-}$,

or, where the areas of the two horizons are merging, i.e.,

$\mathcal{A}_{+}=\mathcal{A}_{-}$,

or, when the entropies of the two horizon are coincident, i.e.,

$\mathcal{S}_{+}=\mathcal{S}_{-}$,

or, when the surface gravities of both horizons are equal, i.e.,

$\kappa_{+}=\kappa_{-}$,

or, when the temperatures of both horizons are the same, i.e.,

$T_{+}=T_{-}$,

or, when the angular velocity of both horizons are coincident, i.e.,

$\Omega_{+}=\Omega_{-}$,

or, when the irreducible masses of both horizons are equal, i.e.,

$\mathcal{M}_{\text {irr+ }}=\mathcal{M}_{\text {irr-- }}$

If any one of the above properties are satisfied then a black hole is said to be an extremal black hole. Thus one gets the area in the extremal limit:

$\mathcal{A}_{+}=\mathcal{A}_{-}=8 \pi \sqrt{J^{2}+\frac{Q^{4}}{4}}$.

As a result of (111), another relation,

$\frac{J^{2}}{\mathcal{M}^{2}}+Q^{2}=\mathcal{M}_{\mathrm{CR}}{ }^{2}$,

of the extremal KN spacetime continues to hold in the presence of the surrounding matter in accordance with the factthat 
$\mathrm{KN}$ black holes are degenerate if they are extremal. $M_{\mathrm{CR}}$ denotes the Christodoulou and Ruffini mass. Also, for the entropy one obtains

$\mathcal{S}_{+}=\mathcal{S}_{-}=2 \pi \sqrt{J^{2}+\frac{Q^{4}}{4}}$.

It is well known that the surface gravity and surface temperature go to zero in the extremal limit, i.e., $\kappa_{+}=\kappa_{-}=0$ and $T_{+}=T_{-}=0$.

It may be noted that the Komar energy goes to zero at the extremal limit, i.e., $E_{+}=E_{-}=0$. This may imply that this is another way of seeing the discontinuity between the extremal spacetime and non-extremal spacetime.

\section{Discussions}

In this work, we have derived the Smarr formula on the Cauchy horizon $\left(\mathcal{H}^{-}\right)$. We have proposed the four laws of black hole mechanics for the inner horizon $\left(\mathcal{H}^{-}\right)$. We have found, in contrast to some earlier work [10,30], particularly for the first law of the inner horizon $\left(\mathcal{H}^{-}\right)$, complete consistency between our results and these results.

We have also demonstrated that the area product, horizon radii product, entropy product, and irreducible mass product of the event horizon and Cauchy horizon are universal, although the surface gravity product, surface temperature product, and the Komar energy product are not universal for a Kerr-Newman black hole.

We have also defined the Christodoulou and Ruffini mass on the Cauchy horizon. We have further showed that the identity $K_{\chi^{\mu}-}=2 \mathcal{S}_{-} T_{-}$is valid on the inner horizon $\left(\mathcal{H}^{-}\right)$ and also the Komar energy in a compact form $E_{-}=2 \mathcal{S}_{-} T_{-}$. This also relates the generalized Smarr formula $E_{-}=\mathcal{M}_{-}$ $2 J \Omega_{-}-Q V_{-}$on $\mathcal{H}^{-}$.

Another interesting point we have found is that the Komar energy goes to zero at the extremal limit, which may display a discontinuity between extremal spacetime and non-extremal spacetime.
Open Access This article is distributed under the terms of the Creative Commons Attribution License which permits any use, distribution, and reproduction in any medium, provided the original author(s) and the source are credited.

Funded by $\mathrm{SCOAP}^{3}$ / License Version CC BY 4.0.

\section{References}

1. L. Smarr, Phys. Rev. Lett. 30, 71 (1973) [Erratum-ibid. 30, 521 (1973)]

2. L. Smarr, Phys. Rev. D 7, 289 (1973)

3. M. Ansorg, J. Hennig, Class. Quant. Grav. 25, 222001 (2008)

4. M. Ansorg, J. Hennig, Phys. Rev. Lett. 102, 221102 (2009)

5. M. Ansorg, J. Hennig, C. Cederbaum, Gen. Rel. Grav. 43, 1205 (2011)

6. F. Larsen, Phys. Rev. D 56, 1005 (1997)

7. M. Cvetic, G.W. Gibbons, C.N. Pope, Phys. Rev. Lett. 106, 121301 (2011)

8. M. Cvetic, F. Larsen, J. High Energy Phys. 09, 088 (2009)

9. B. Chen, S.X. Liu, J.J. Zhang, J. High Energy Phys. 1211, 017 (2012)

10. A. Castro, M.J. Rodriguez, Phys. Rev. D 86, 024008 (2012)

11. M. Visser, Phys. Rev. D 88, 044014 (2013)

12. V. Faraoni, A.F.Z. Moreno, Phys. Rev. D 88, 044011 (2013)

13. M. Visser, J. High Energy Phys. 06, 023 (2012)

14. S. Chandrashekar, The Mathematical Theory of Black Holes (Clarendon Press, Oxford, 1983)

15. D. Christodoulou, R. Ruffini, Phys. Rev. D 4, 3552 (1971)

16. E. Newman, K. Chinnaparad, A. Exton, A. Prakash, R. Torrence, J. Math. Phys. 6, 918 (1965)

17. R.P. Kerr, Phys. Rev. Lett. 11, 237 (1963)

18. J.D. Bekenstein, Phys. Rev. D 7, 2333 (1973)

19. J.D. Bekenstein, Phys. Rev. D 9, 3292 (1974)

20. A. Komar, Phys. Rev. 113, 934 (1959)

21. P.C.W. Davies, Rep. Prog. Phys. 41 (1978)

22. D. Christodoulou, Phys. Rev. Lett. 25, 1596 (1970)

23. D. Bini, R. Jantzen, R. Ruffini, Reinterpretation of the Mass Formula for Black Hole (2012)

24. R. Penrose, R.M. Floyd, Nature 229, 177 (1971)

25. R. Penrose, Riv. Nuovo Cimento 1, 252 (1969)

26. J.M. Bardeen, B. Carter, S.W. Hawking, Commun. Math. Phys. 31, 161 (1973)

27. S. Modak, S. Samanta, Int. J. Theor. Phys. 51, 1416 (2012)

28. T. Padmanabhan, Class. Quant. Grav. 21, 4485 (2004)

29. R. Banerjee, B.R. Majhi, Phys. Rev. D 81, 124006 (2010)

30. S. Detournay, Phys. Rev. Lett. 109, 031101 (2012) 